\title{
Mining R Coronae Borealis stars from Catalina surveys (Research Note)
}

\author{
C.-H. Lee (李見修) ${ }^{1,2}$ \\ ${ }^{1}$ University Observatory Munich, Scheinerstrasse 1, 81679 Munich, Germany \\ e-mail: chlee@usm.lmu.de \\ 2 Max Planck Institute for Extraterrestrial Physics, Giessenbachstrasse, 85748 Garching, Germany
}

Received 19 July 2014 / Accepted 19 December 2014

\begin{abstract}
Aims. R Coronae Borealis stars (RCBs) are rare carbon stars that lack of hydrogen in their photospheresand are most likely products of white dwarf mergers. A census of RCBs can shed light on the progenitors of SNe Ia in the context of a double degenerate scenario. Methods. Traditionally, RCBs are identified by their unpredictable photometric variation with dimmings up to 9 mag, and thus discoveries of RCBs are heavily biased to the limited regions monitored by long-term microlensing experiments. However, recent studies of galactic RCBs have shown that they exhibit distinct mid-infrared colors originating from their cool circumstellar shells, and the all-sky WISE survey facilitates the identification of RCB candidates. Therefore, combining the WISE colors with large area time-domain surveys will enable us to discover more RCBs.

Results. This study presents the results of $26 \mathrm{RCB}$ candidates from the Catalina surveys, where five of them are spectroscopically confirmed RCBs and seven of them are previously known carbon stars. This demonstrates the efficacy of this kind of an approach and the potential to discover uncharted RCBs in ongoing and future synoptic surveys.
\end{abstract}

Key words. circumstellar matter - stars: carbon

\section{Introduction}

$\mathrm{R}$ Coronae Borealis (RCB) stars are hydrogen deficient, carbon rich supergiants that exhibit aperiodic dimmings up to 9 mag in the $V$-band (Clayton 2012). These dimmings are caused by a sudden formation of carbon dust close to the line-of-sight that obscures the photosphere of the star. Despite their intrinsically high brightness ( $-5 \leq M_{V} \leq-3.5$, Tisserand et al. 2009), only 76 Galactic RCBs (Tisserand et al. 2013), 22 RCBs in the Magellanic Clouds (see Clayton 2012, and references therein), and 2 M31 RCBs (Tang et al. 2013) have been discovered to date. This might be because of the lack of all-sky, long-term monitoring programs to witness their sudden drop in brightness and/or the fact that RCBs represent a very brief phase of stellar evolution.

The deficiency of hydrogen and enrichment of carbon in the atmosphere of RCBs suggest that they are at a final stage of stellar evolution. Two scenarios have been proposed to explain the properties of RCBs: the white dwarf merger scenario (Webbink 1984) and the final helium shell flash scenario (FF, Iben et al. 1996). In the first scenario, RCBs are regarded as products of a carbon-oxygen white dwarf merged with a helium white dwarf, commonly dubbed the double degenerate scenario (DD). In the latter scenario, a star expands rapidly to supergiant size shortly before turning into a white dwarf. On the one hand, observations of ${ }^{18} \mathrm{O}$ overabundances in cool RCBs (Clayton et al. 2007), as well as surface abundance anomalies, especially fluorine (Pandey et al. 2008), strongly support the DD scenario. On the other hand, discoveries of four RCBs associated with nebulae (Clayton et al. 2011) indicate that part of the RCBs can originate from the FF scenario. The DD scenario is of special interest because it is a low-mass analog of SNe Ia formed via the DD channel. Hence, studies of RCBs can provide insights to the progenitors to $\mathrm{SNe}$ Ia.

Because of their sudden fading and hence the need for long-term monitoring, previous discoveries of RCBs are limited and are heavily biased to microlensing surveys (e.g., Alcock et al. 2001; Tisserand et al. 2009; Soszyński et al. 2009) or dedicated monitoring of local luminous fields (Tang et al. 2013). With the advance in studies of infrared signatures of RCBs (Tisserand 2012), as well as the all-sky automated survey (ASAS, Pojmanski 2002), the number of RCBs has increased dramatically in the past years (Tisserand et al. 2013; Miller et al. 2012). However, ASAS is limited to $V \sim 13$, while RCBs can be fainter. This motivates us to search for RCBs in the Catalina Surveys (Drake et al. 2009), which reach to $\sim 20 \mathrm{mag}$ in $V$, and extend the ASAS to fainter magnitudes.

This paper is organized as follows. In Sect. 2 we briefly describe the data used in this study. The methods used to search for RCBs are presented in Sect. 3. The selected candidates are discussed in Sect. 4, followed by a summary and outlook in Sect. 5.

\section{Data source}

To witness the aperiodic photometric variations of candidate $\mathrm{RCBs}$, we incorporate data from the long-term, large-area Catalina surveys. Started in 2004, the Catalina surveys employ three different telescopes to constantly patrol the sky between $75<$ Dec $<70 \mathrm{deg}$, with the goal of searching for near- Earth objects and potentially hazardous asteroids. Each of the three 
telescopes is operated as a separate sub-survey. These include the $0.7 \mathrm{~m}$ Catalina Schmidt Telescope, equipped with a 8-deg ${ }^{2}$ fieldof-view (FOV) camera, the $1.5 \mathrm{~m}$ Mount Lemmon Telescope, equipped with a $1-\mathrm{deg}^{2}$ FOV camera, which are both located in Arizona, USA, as well as the $0.5 \mathrm{~m}$ Siding Spring Telescope, equipped with a 4.2- $\mathrm{deg}^{2}$ FOV camera in Australia. All three telescopes survey their own patches of the sky, and are 10-15 deg off the Galactic plane to avoid crowded stellar regions. All images are taken without filters to maximize the throughput. The observations are normally taken in sets of four images separated by $10 \mathrm{~min}$, with typical exposure times up to $30 \mathrm{~s}$. Photometry is carried out with SExtractor (Bertin \& Arnouts 1996) based on aperture photometry. In addition to the search for asteroids, all the Catalina surveys data are analyzed for transient sources by the Catalina Real-time Transient Survey (CRTS, Drake et al. 2009). This work is based on the Catalina surveys data release 2 (CSDR2), which provides photometry for 500 million objects in the region of $-75<$ Dec $<70 \mathrm{deg}$ and $|b|>15 \mathrm{deg}$.

\section{Candidate selection}

Tisserand (2012) has established a procedure to select candidate RCBs using their distinct infrared colors from 2MASS (Skrutskie et al. 2006) and WISE (Wright et al. 2010). The faintest known RCB, EROS-LMC-RCB-5 in the Large Magellanic Cloud, is cataloged in both WISE and 2MASS surveys. This indicates that we are able to identify potential Galactic RCBs within $50 \mathrm{kpc}$ with the WISE and 2MASS data if they are lightly reddened.

In addition to the infrared color selections, RCBs are characterized by their aperiodic dimmings. Previous searches utilized ASAS (Tisserand et al. 2013), which were limited to $V \sim 14$ mag. In this work, we make use of the Catalina surveys (Drake et al. 2009) to extend the search to the faint end (up to $V \sim 20 \mathrm{mag}$ ). A constraint of $\Delta V>1 \mathrm{mag}$ is added on top of the infrared selection criteria to identify sources exhibiting photometric variations. In the end, we obtain a sample of 26 RCB candidates. Their photometry is listed in Table 1, and their finding charts, light curves, and spectral energy distributions are shown in Fig. 1-3, respectively. Further discussions on their properties are given in the following section.

\section{Discussion}

\subsection{Candidates in the literature}

We queried the SIMBAD database ${ }^{1}$ to see whether our candidates are known RCBs or attributed to other types of variability. The type assignation of each candidate from SIMBAD is listed in Table 1 . Five of our candidates are spectroscopically confirmed RCBs (highlighted with bold font in Table 1), but only three of them are shown as known-RCBs in the SIMBAD database, where UX Ant (J105709.05-372355.) and U Aqr (J220319.69163735.0) have long been established as RCB stars Clayton (1996), while the RCB nature of ASAS-RCB-14 (J164729.74152522.9) has recently been revealed thanks to the ASAS survey (Tisserand et al. 2013). For the remaining two, AO Her is assigned as a semiregular variable. However, recent photometric studies from AAVSO and spectroscopic information point out that this is a bona fide RCB (see Otero et al. 2014, and reference therein). NSV 11154 is assigned a long-period variable in SIMBAD. However, light curves of NSV 11154 extracted

\footnotetext{
1 http://simbad.u-strasbg.fr/simbad/
}

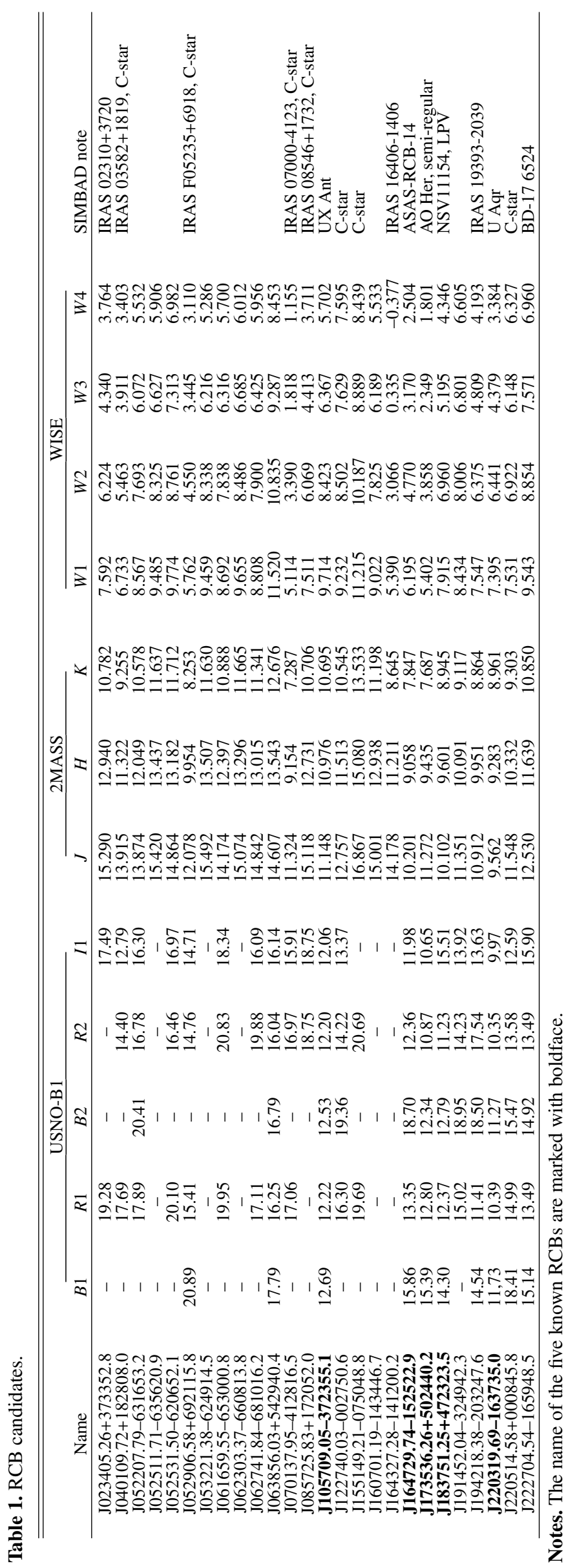




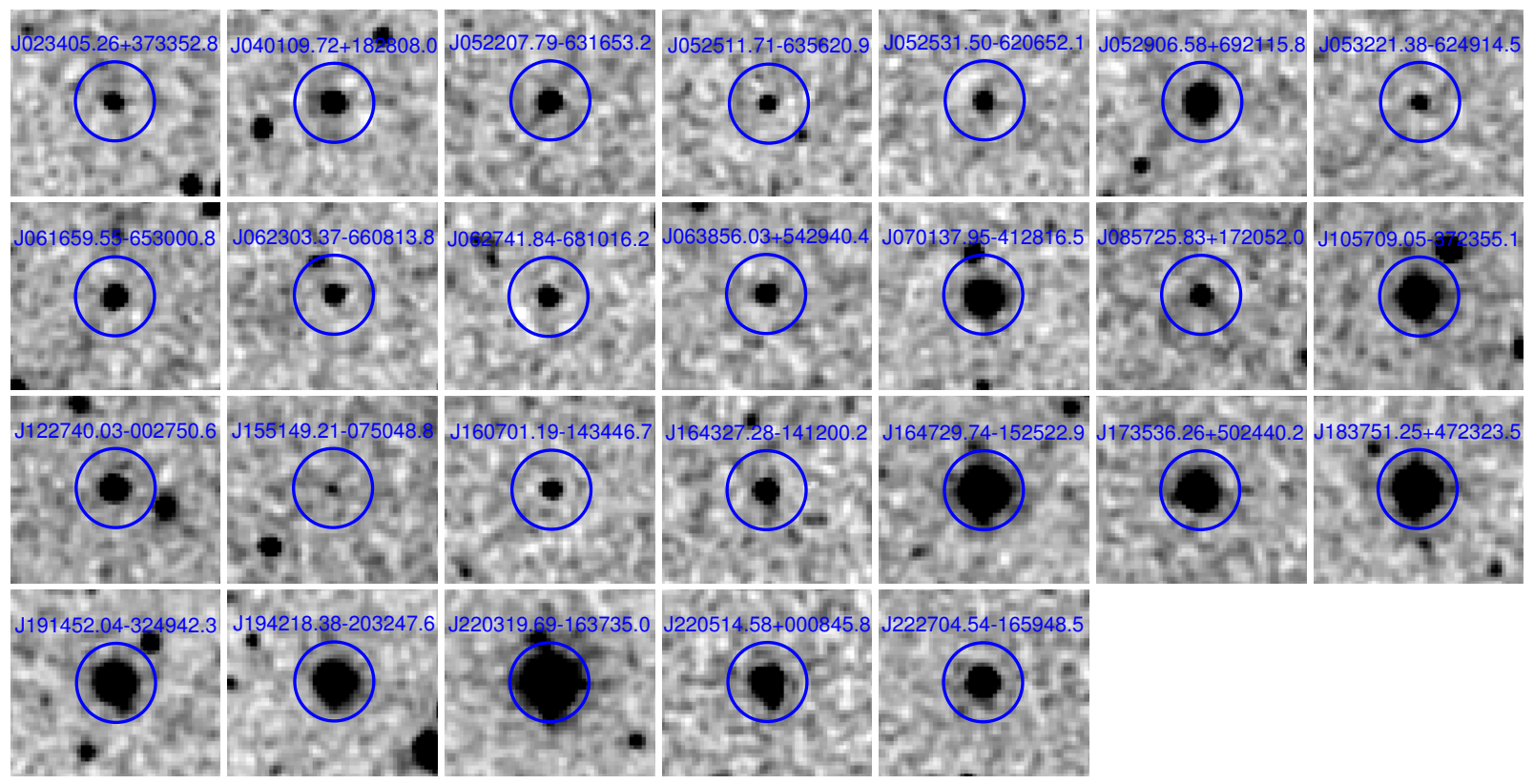

Fig. 1. Finding charts of the 26 candidates. The underlying images are taken from the 2MASS $J$-band. The position of each candidate is outlined with a circle with $10^{\prime \prime}$ radius.

from the ROTSE-I Northern Sky Variability Survey Akerlof et al. (2000), the Sonnenberg Plate data (Haussler et al. 2009) dated back to 1964-1996 and the Harvard College Observatory plate archive processed by the Digital Access to a Sky Century at Harvard (DASCH) program Grindlay et al. (2009), as well as additional observations from AAVSO's Sonoita Research Observatory and spectra taken with the $4 \mathrm{~m}$ telescope at KPNO (Kijbunchoo et al. 2011) indicate that NSV 11154 is a bona fide RCBtype star.

Besides these five known RCBs, another seven candidates are listed as carbon stars from the SIMBAD database, which indicates that the selection procedure effectively identifies carbon stars with distinct infrared signatures and photometric variations that are plausible RCB stars. However, three out of these seven candidates had spectroscopic observations and are categorized as N-type AGB stars, i.e., J085725.83+172052.0 (Groenewegen et al. 1997), J122740.03-002750.6 (Totten \& Irwin 1998), and J220514.58+000845.8 (Mauron et al. 2004). This suggests that a fraction of the candidates is contaminated by the $\mathrm{N}$-type AGB stars.

\subsection{Spectral energy distribution}

The difference between the N-type AGB stars and RCB stars is that the former are normally cooler $(T \sim 3000 \mathrm{~K}$, Tanaka et al. 2007), while the later are hotter $(T \sim 4000-8000)$ and have a shell with a temperature between 400-1000 K (Tisserand 2012). Hence, a possible means to distinguish between N-type AGB stars and RCB stars is through the shape of the spectral energy distribution (SED), where N-type AGB stars show a onecomponent SED that peaks in the infrared (e.g., see Fig. 3 of Groenewegen et al. 1997), while the RCBs exhibit a SED with one component that peaks at optical wavelengths superposed with another component that peaks in the infrared (see, e.g., Fig. 9 of Tisserand 2012).

To improve the wavelength coverage in the optical, we make use of the USNO-B1 photometry (Monet et al. 2003). The USNO-B1 catalog has two measurements in $B$ and $R$ filters, and one measurement in $I$-filter. Since RCBs vary their magnitude by up to 9 mag in the optical, the two measurements in $B(R)$ are compared, and we only adopt the brighter measurement in $B(R)$ in the SED analysis. However, it is not guaranteed that the brighter measurement is the true brightness at light curve maximum, which will impact the interpretation of the SED as well.

The positions of the 26 candidates are matched with the USNO-B1 catalog to find optical counterparts of the infrared selected sources. However, only 21 have USNO-B1 matches within 10 arcsec. The other five might be intrinsically faint in optical filters, or were in the dimming phases when the USNO-B1 observations were taken.

We show the SEDs of these 26 candidates in Fig. 3. The five spectroscopically confirmed RCBs have SEDs that can be interpreted as having two components, except ASAS-RCB-14 (J164729.74-152522.9), which has a SED dominated by one component. This is a case where the USNO-B1 measurements were taken during the dimming phases, and the stellar component is heavily obscured by the carbon cloud formed close to the line of sight.

\subsection{Plausible RCBs from light curves and SEDs}

RCB stars have unique light curves (see, e.g., Clayton 2012). They stay at maximum brightness for hundreds to thousands of days, with intermittent sharp declines that drop more than three magnitudes within days or weeks, followed by slower recoveries (months to years) to the maximum brightness. After examining the light curves of the 26 selected candidates presented in Sect. 3 , we found five light curves exhibiting the unique features of RCB light curves. These are J105709.05-372355.1, J164729.74152522.9, J173536.26+502440.2, J183751.25+472323.5, and J220319.69-163735.0. All of them are already known RCBs (see Sect. 4.1). Besides these five RCBs, J194218.38-203247.6 also shows brightness variations $>3 \mathrm{mag}$, though from its light curve shape it is not certain to be a RCB. In addition, there are two more candidates, J062741.84-681016.2 and J160701.19143446.7, which could have been in decline. Their light curves during the Catalina surveys could be the slow recovery part of 

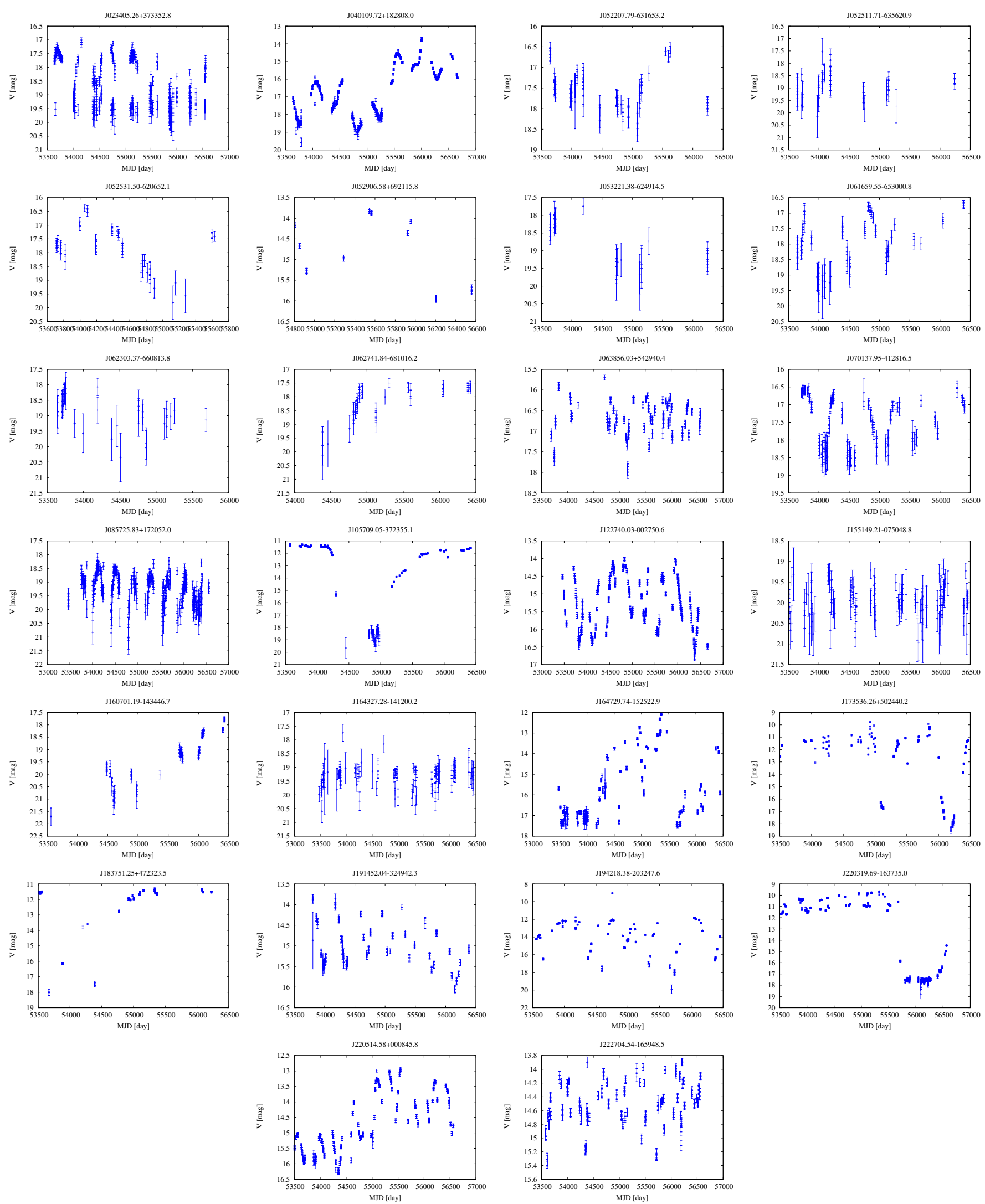

Fig. 2. $V$-band light curves of the 26 selected RCB candidates from CSDR2.

typical RCBs, though their light curves only show monotonously increasing in brightness and are not very informative. We further examined the SEDs of the five known RCBs and the three additional objects to verify/disprove their candidacies.
As shown by Tisserand (2012), the SEDs of RCBs can be interpreted as two components: i) a hotter component with $T \sim 4000-8000 \mathrm{~K}$ originates from the central star and peaks at the optical wavelength; and ii) a cooler component with 
C.-H. Lee: Mining R Coronae Borealis stars from Catalina surveys $(R N)$
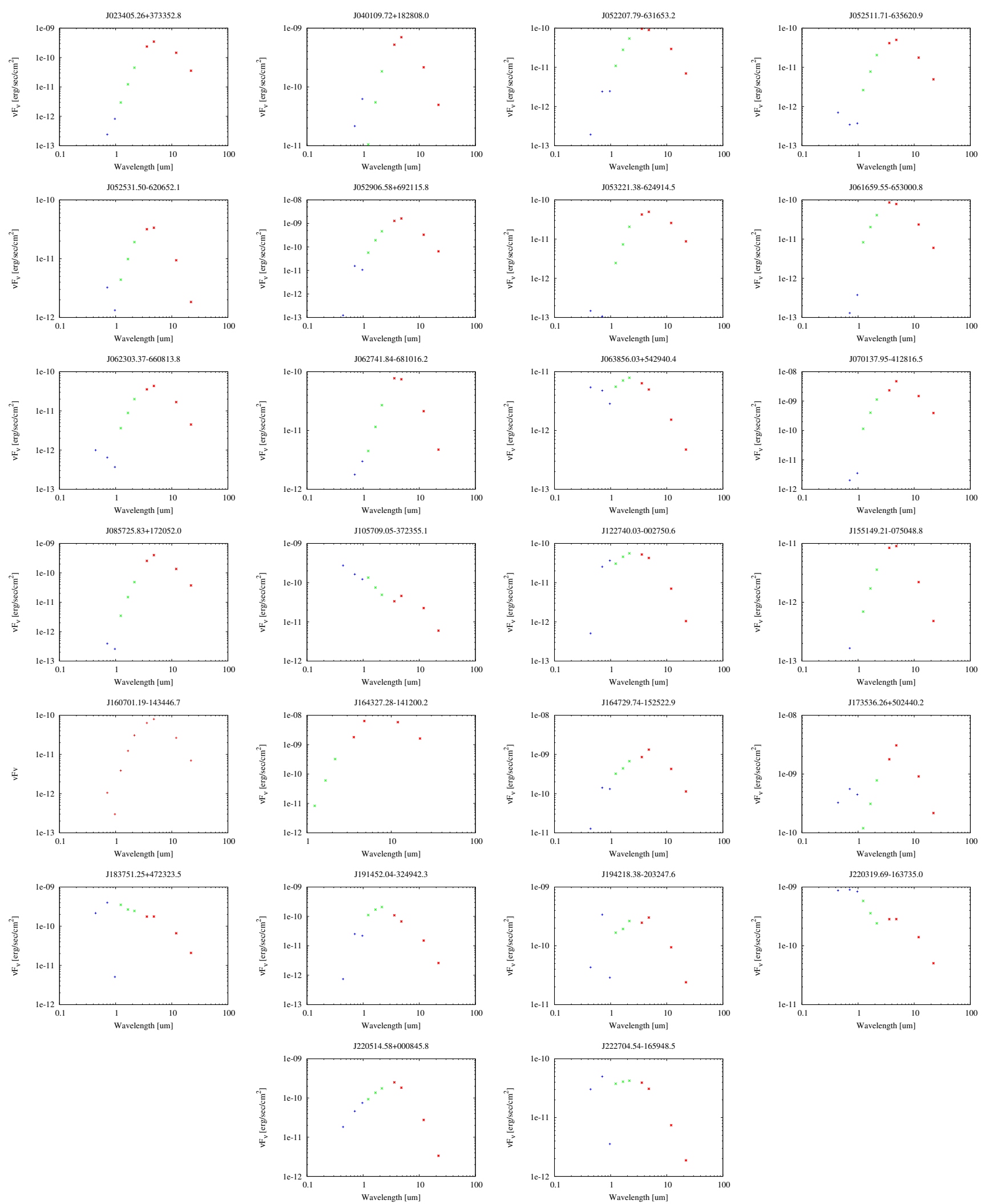

Fig. 3. Spectral energy distribution of 26 RCB candidates from the photometry of USNO-B1 (blue), 2MASS (green), and WISE (red).

$T \sim 400-1000 \mathrm{~K}$ contributed by the circumstellar shell and peaks at the infrared wavelength. After examining the SEDs of the aforementioned eight objects, we found that all of the five spectroscopically confirmed RCBs, except J164729.74152522.9, indeed show these two components in their SEDs.
One possible explanation is that the optical (USNO-B1) photometry of J164729.74-152522.9 was taken during the dimming phases, and the stellar component is heavily obscured by the carbon cloud. 
For the other three objects, there is no spectroscopic information in the literature. The object J194218.38-203247.6 has a relatively flat SED between 1 and $10 \mu \mathrm{m}$, hints at a SED composed of two components, which supports its RCB candidacy. We encourage spectroscopic follow-up to verify/disprove it as a RCB. The object J062741.84-681016.2 has a one-component SED peaks at the infrared, hence does not support the RCB interpretation. The object J160701.19-143446.7 has no detection in the USNO-B1, hence we could not verify/disprove its candidacy via SED.

\section{Summary and prospects}

Following the procedure of Tisserand (2012), we select plausible RCB candidates from their infrared colors documented in the 2MASS and WISE catalogs. These candidates are cross-matched to the Catalina surveys, which yield light curves spanning a period of seven years. Based on the light curve variation, $26 \mathrm{RCB}$ candidates are selected, among which five are spectroscopically confirmed RCBs, and seven candidates are cataloged as carbon stars in the SIMBAD database.

The limitation of the Catalina surveys is that they only cover regions with Galactic latitude $|b|>15 \mathrm{deg}$ to avoid crowded stellar regions, especially the Galactic plane. However, the vast majority of the WISE-selected candidates reside in the Galactic plane (Tisserand 2012). Therefore, the Catalina surveys only contain light curves for a small fraction of the infrared select candidates. Ongoing and future surveys, such as Palomar Transient Factory (Law et al. 2009; Rau et al. 2009), PanSTARRSS (Kaiser et al. 2010), SkyMapper (Keller et al. 2007), and LSST (LSST Science Collaboration et al. 2009), will deliver time-series photometry for sources at low Galactic latitude, and hopefully can give us a complete picture of the Galactic RCB population.

Acknowledgements. This work was supported by the DFG cluster of excellence Origin and Structure of the Universe (http://www . universe-cluster.de). This publication makes use of data products from the Two Micron All Sky Survey, which is a joint project of the University of Massachusetts and the Infrared Processing and Analysis Center/California Institute of Technology, funded by the National Aeronautics and Space Administration and the National Science Foundation. This publication makes use of data products from the Wide-field Infrared Survey Explorer, which is a joint project of the University of California, Los Angeles, and the Jet Propulsion Laboratory/California Institute of Technology, funded by the National Aeronautics and Space Administration. The
CSS survey is funded by the National Aeronautics and Space Administration under Grant No. NNG05GF22G issued through the Science Mission Directorate Near-Earth Objects Observations Program. The CRTS survey is supported by the US National Science Foundation under grants AST-0909182 and AST-1313422.

\section{References}

Akerlof, C., Amrose, S., Balsano, R., et al. 2000, AJ, 119, 1901 Alcock, C., Allsman, R. A., Alves, D. R., et al. 2001, ApJ, 554, 298 Bertin, E., \& Arnouts, S. 1996, A\&AS, 117, 393

Clayton, G. C. 1996, PASP, 108, 225

Clayton, G. C. 2012, J. American Association of Variable Star Observers (JAAVSO), 40, 539

Clayton, G. C., Geballe, T. R., Herwig, F., Fryer, C., \& Asplund, M. 2007, ApJ, 662, 1220

Clayton, G. C., Sugerman, B. E. K., Stanford, S. A., et al. 2011, ApJ, 743, 44

Drake, A. J., Djorgovski, S. G., Mahabal, A., et al. 2009, ApJ, 696, 870

Grindlay, J., Tang, S., Simcoe, R., et al. 2009, Preserving Astronomy's Photographic Legacy: Current State and the Future of North American Astronomical Plates, 410, 101

Groenewegen, M. A. T., Oudmaijer, R. D., \& Ludwig, H.-G. 1997, MNRAS, 292,686

Haussler, K., Berthold, T., \& Kroll, P. 2009, Inform. Bull. Variable Stars, 5890, 1 Høg, E., Fabricius, C., Makarov, V. V., et al. 2000, A\&A, 355, L27

Iben, I., Jr., Tutukov, A. V., \& Yungelson, L. R. 1996, ApJ, 456, 750

Kaiser, N., Burgett, W., Chambers, K., et al. 2010, Proc. SPIE, 7733,

Keller, S. C., Schmidt, B. P., Bessell, M. S., et al. 2007, PASA, 24, 1

Kijbunchoo, N., Clayton, G. C., Vieux, T. C., et al. 2011, PASP, 123, 1149

Law, N. M., Kulkarni, S. R., Dekany, R. G., et al. 2009, PASP, 121, 1395

LSST Science Collaboration, Abell, P. A., Allison, J., et al. 2009 [arXiv:0912.0201]

Masci, F. J., \& Fowler, J. W. 2009, Astronomical Data Analysis Software and Systems XVIII, 411, 67

Mauron, N., Azzopardi, M., Gigoyan, K., \& Kendall, T. R. 2004, A\&A, 418, 77

Miller, A. A., Richards, J. W., Bloom, J. S., et al. 2012, ApJ, 755, 98

Monet, D. G., Levine, S. E., Canzian, B., et al. 2003, AJ, 125, 984

Otero, S., Hümmerich, S., Bernhard, K., \& Sozynski, I. 2014, J. American Association of Variable Star Observers (JAAVSO), 42, 13

Pandey, G., Lambert, D. L., \& Rao, N. K. 2008, ApJ, 674, 1068

Pojmanski, G. 2002, Acta Astron., 52, 397

Rau, A., Kulkarni, S. R., Law, N. M., et al. 2009, PASP, 121, 1334

Skrutskie, M. F., Cutri, R. M., Stiening, R., et al. 2006, AJ, 131, 1163

Soszyński, I., Udalski, A., Szymański, M. K., et al. 2009, Acta Astron., 59, 335

Tanaka, M., Letip, A., Nishimaki, Y., et al. 2007, PASJ, 59, 939

Tang, S., Cao, Y., Bildsten, L., et al. 2013, ApJ, 767, L23

Tisserand, P. 2012, A\&A, 539, A51

Tisserand, P., Wood, P. R., Marquette, J. B., et al. 2009, A\&A, 501, 985

Tisserand, P., Clayton, G. C., Welch, D. L., et al. 2013, A\&A, 551, A77

Totten, E. J., \& Irwin, M. J. 1998, MNRAS, 294, 1

Webbink, R. F. 1984, ApJ, 277, 355

Wright, E. L., Eisenhardt, P. R. M., Mainzer, A. K., et al. 2010, AJ, 140, 1868 